\title{
GaSb DOTS GROWN ON GaAs SURFACE BY METALORGANIC CHEMICAL VAPOUR DEPOSITION*
}

R. Bożek, A. Babiński, J.M. Baranowski, R. StęPNiewski

Institute of Experimental Physics, Warsaw University

Hoża 69, 00-681 Warszawa, Poland

Z. KLuSeK, W. Olejniczak

Department of Solid State Physics, University of Łódź

Pomorska 149/153, 90-236 Eódź, Poland

K. STAROWIEYSKi

Department of Chemistry, Technical University of Warsaw

Koszykowa 75, 00-662 Warszawa, Poland

AND J. WRÓBEL

Institute of Physics, Polish Academy of Sciences

Al. Lotników 32/46, 02-668 Warszawa, Poland

We report metaloorganic chemical vapour deposition growth of an anisotropic $\mathrm{GaSb}$ islands on $\mathrm{GaAs}(001)$ surface with a typical dimensions around $200 \mathrm{~nm}$. Results of investigations employing scanning electron microscope, scanning tunnelling microscope and photocapacitance are presented.

PACS numbers: 68.55.-a, 81.15.Gh

Epitaxial growth of lattice mismatched heterostructures has been extensively investigated for many different systems. Strained epitaxial layers are unstable and after depositing few monolayers undergo transition from 2D to 3D growth mode. Such process helps to relieve strain arising from difference in lattice constants between the layer and the substrate and leads to formation of three-dimensional islands. Resulting structures are considered as technologically promising due to a lower density of defects comparing to their lithographically prepared counterparts. Moreover, understanding of initial steps of heteroepitaxial growth has crucial meaning for obtaining high quality epilayers.

*This work was partially supported by the State Committee for Scientific Research (Republic of Poland) grant no. PBZ-101-01. 
In the following paper we present results concerning $\mathrm{GaSb}$ on $\mathrm{GaAs}$ system. Lattice constants of GaAs and GaSb are respectively $5.65 \AA$ and $6.09 \AA$. These give relative lattice mismatch of $7.8 \%$, which is much higher than in the case of $\mathrm{Ge}$ on $\mathrm{Si}$ or GaInAs on GaAs, which are more often investigated systems. So far results for thin GaSb layers grown on GaAs were obtained for MBE grown samples [1, 2].

The investigated layers were grown in atmospheric pressure metaloorganic chemical vapour deposition (MOCVD) reactor with horizontal, RF-heated graphite susceptor. The growth of all discussed samples was performed at temperature of $575^{\circ} \mathrm{C}$ with trimethylgallium and trimethylantimony as $\mathrm{Ga}$ and $\mathrm{Sb}$ sources. The noncommercial metaloorganic compounds were synthetized by methods designed at Warsaw Technical University. The substrates were (001) oriented GaAs wafers. To improve surface quality, the GaAs buffer with thickness of $200 \mathrm{~nm}$ was grown prior to the $\mathrm{GaSb}$ deposition. The nominal thickness of the GaSb layers was in the range from 60 to $120 \AA$ (growth rate was calibrated for $1 \mu \mathrm{m}$ thick layer), which corresponds to 20-40 monolayers. For comparison we grew the reference sample consisting only of a GaAs buffer. We also investigated how the growth of the islands is influenced by initially strained buffers including 5 periods consisting of $60 \AA \mathrm{GaSb}$ and $100 \AA \mathrm{GaAs}$ layers. Such a structure should cause formation of the islands in the buried layers and thus enhance nucleation on the surface. Similar process was observed during growth of self-organised quantum disks in the multilayer InGaAs-GaAlAs system [3].

Images received from the scanning electron microscope (SEM) revealed presence of islands with an average density of $4 \times 10^{8} \mathrm{~cm}^{-2}$ (Fig. 1). The islands are ellipsoidal with a long axis parallel to the (110) direction. Typical lateral size (long axis) of the islands in the case of $60 \AA$ thick layer grown on unstrained GaAs buffer is found to be of order of $2000 \AA$. The aspect ratio is around 3. The
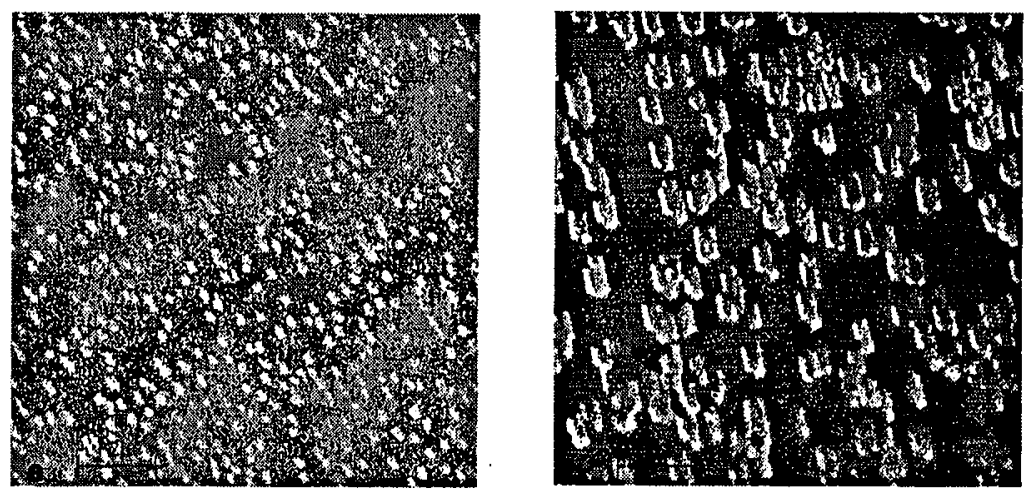

Fig. 1. SEM micrographs of GaSb island formed (a) on the initially unstrained GaAs buffer after depositing nominally 20 monolayers $\mathrm{GaSb}$ and (b) on the strained buffer after depositing of 40 monolayers $\mathrm{GaSb}$. Note that total area covered with the islands is not proportional to the amount of deposited material but depends on the strain in the buffer. 
scanning tunnelling microscope (STM) measurements allowed to determine the characteristic height of the islands, which exceeds $1000 \AA$. The height comparable with the lateral dimensions is an argument for strongly three-dimensional growth mode. Figure $1 \mathrm{~b}$ shows islands formed on the strained buffer after deposition of nominally $120 \AA \mathrm{GaSb}$ layer. It is visible that the total area covered with islands increases much faster than the amount of deposited material.

It is theoretically predicted [4] that the $\mathrm{GaSb} / \mathrm{GaAs}$ system is a type II heterostructure with $\mathrm{GaSb}$ conduction band about $0.5 \mathrm{eV}$ above $\mathrm{GaAs}$ conduction band. It reduces the chance of observing photoluminescence for such a system. However, the existence of GaSb islands on GaAs buffer layer can be observed by means of electrical methods. Using the photovoltaic spectroscopy (PVS) option of the Bio-rad Electrochemical Profiler we measured a photovoltaic response of the material on illumination. We observed a broad band in PVS spectrum in the region of 1.0-1.4 $\mu \mathrm{m}$. There was no structure of PVS spectrum which could be attributed to carrier confinement. This signal disappeared after removing nominally $5 \mathrm{~nm}$ of the material. Moreover, no such a band was found on PVS spectra measured on a reference sample.

We report results of technological studies of $\mathrm{GaSb}$ islands grown for the first time by atmospheric pressure MOCVD. The existence of $\mathrm{GaSb}$ islands has been directly observed by SEM and STM.

\section{References}

[1] J.M. Kang, Suk-Ki Min, A. Rocher, Appl. Phys. Lett. 65, 2954 (1994).

[2] B. Brar, D. Leonard, Appl. Phys. Lett. 66, 462 (1995).

[3] R. Nötzel, J. Temmyo, T. Tammamura, Nature 369, 131 (1994). 